\title{
Erratum to: Application of a newly developed column test device to analyze seawater transport in sandy soils
}

\author{
Xiaoqiang Dong ${ }^{1,2,4} \cdot$ Yongsung $\mathrm{Kim}^{3} \cdot$ Heesoo Woo ${ }^{4}$ Hisoo Park ${ }^{4}$. \\ Junboum Park ${ }^{4}$
}

Published online: 18 April 2015

(c) Springer-Verlag Berlin Heidelberg 2015

\section{Erratum to: Environ Earth Sci (2013) \\ 70:2397-2404 \\ DOI 10.1007/s12665-013-2675-6}

The authors would like to correct errors in the original publication as detailed below.

The correct version of the author lists is given here:

Xiaoqiang Dong · Yongsung Kim · Heesoo Woo ·

Hisoo Park · Junboum Park

X. Dong $(\square)$

College of Architecture and Civil Engineering,

Taiyuan University of Technology, Taiyuan 030024, China

e-mail: dxqtyut@yahoo.com; dongxiaoqiang@126.com

\section{Dong}

Key Laboratory of Coal Science and Technology, Ministry of Education and Shanxi Province, Taiyuan 030024, China
Y. Kim

Knowledge Development and Management, Global Green Growth Institute (GGGI), Seoul, South Korea

X. Dong $\cdot$ H. Woo $\cdot$ H. Park - J. Park

Department of Civil and Environmental Engineering, Seoul National University, Seoul 151-742, South Korea

The online version of the original article can be found under doi: 10.1007/s12665-013-2675-6.

Xiaoqiang Dong

dxqtyut@yahoo.com; dongxiaoqiang@126.com

1 College of Architecture and Civil Engineering, Taiyuan University of Technology, Taiyuan 030024, China

2 Key Laboratory of Coal Science and Technology, Ministry of Education and Shanxi Province, Taiyuan 030024, China

3 Knowledge Development and Management, Global Green Growth Institute (GGGI), Seoul, South Korea

4 Department of Civil and Environmental Engineering, Seoul National University, Seoul 151-742, South Korea 